This is the author's version of a work that was accepted for publication in Complementary Therapies in Clinical Practice. Changes resulting from the publishing process, such as peer review, editing, corrections, structural formatting, and other quality control mechanisms may not be reflected in this document. Changes may have been made to this work since it was submitted for publication. A definitive version was subsequently published as Bishop FL, Lim CY, Leydon G, Lewith GT. (2009) Overseas Chinese students in the UK: Patterns and correlates of their use of Western and Traditional Chinese Medicine. Complementary Therapies in Clinical Practice, 15(1), 813. doi: 10.1016/j.ctcp.2008.09.005. Epub2008 Dec 2. 


\section{Overseas Chinese students in the UK: Patterns and Correlates of their Use of Western and Traditional Chinese Medicine}

Keywords: Medicine, Chinese Traditional; Health Knowledge, Attitudes, Practice; Questionnaires; Student Health Services; Health Services Accessibility Authors: Felicity L Bishop, Chiw Yeh Lim, Geraldine M. Leydon, George T Lewith. Institution: Department of Primary Medical Care, School of Medicine, University of Southampton, UK.

Corresponding author: Dr Felicity L Bishop. Department of Primary Medical Care, School of Medicine, University of Southampton, Aldermoor Health Centre, Aldermoor Close, Southampton, HANTS, UK SO16 5ST. Telephone: +44 (0)2380 241072 Fax: +44 (0)2380 701125 Email: F.L.Bishop@ southampton.ac.uk

\section{Acknowledgements}

We would like to thank all the students who participated in this study and See Ying Hoe who completed the back translation.

\section{$\underline{\text { Conflicts of Interest }}$}

We have no conflicts of interest.

\section{Role of the funding source}

At the time of this work Dr Bishop was supported by a grant from the Southampton Complementary Medicine Research Trust. Ms Lim is a medical student at the University of Southampton. Dr Leydon's post is funded by the University of Southampton. Dr Lewith's post is funded by a grant from the Maurice Laing Foundation. None of our funders had any role in the study design, procedures, or reporting. 


\begin{abstract}
We explored the correlates of use of TCM and WM by ethnic Chinese students in the UK. A questionnaire assessed key theoretical determinants of health services use. 170 ethnic Chinese participants (international students at one university in the South of England) completed this questionnaire (presented in English and Chinese) assessing their demographic characteristics, health status, attitudes towards and use of TCM and WM. Participants were more likely to use WM than TCM when they were in the UK. Different variables predicted use of WM and TCM. The statistical predictors (demographic characteristics, health status, past behaviour, attitudes) explained modest but important proportions of the variance in use of WM (37\%) and TCM (29\%). In conclusion, this small exploratory study suggests a need for further research on the health care utilisation of this growing body of international students. Improved language support is needed for international students in UK health care settings.
\end{abstract}




\section{INTRODUCTION}

In the UK Traditional Chinese Medicine (TCM) is an alternative to conventional, Western Medicine (WM) for both the indigenous and immigrant populations. Although TCM and WM are based on fundamentally different ideas about health and illness, evidence suggests that Chinese migrants in the UK use both systems of health care ${ }^{1 ; 2}$. TCM use is also highly prevalent among Chinese immigrants in the US ${ }^{3}$. It is important to understand how and why Chinese people living in the UK access different health care systems, both to inform policy development at local and national levels and to improve theoretical understandings of patients' health care behaviours. This study explores the correlates of TCM and WM use among a growing sub-group of the Chinese population in the UK, ethnic Chinese international students studying at a British university. This population has not been well-studied, and the small literature that does exist on Chinese migrants' use of health services focuses on permanent, not temporary, migrants ${ }^{2}$ and/or on sub-groups such as women ${ }^{1}$ or community elders ${ }^{4}$.

We used Andersen's revised socio-behavioural model ${ }^{5}$ to determine which theoretically likely correlates of TCM and WM use should be included in this exploratory study. The original version of this model ${ }^{6}$ has been used extensively to study access to and use of conventional health care services, but has been criticized for a perceived lack of attention to health outcomes, an undue focus on individual-level factors to the detriment of social factors, and a static rather than dynamic view of health care utilisation $^{7}$. In response to these and other critiques, Andersen revised the model to better reflect the dynamic nature of health services use, to encompass health outcomes, and to better represent the social nature of many of the central constructs in the model (including 
perceived need for care) ${ }^{5}$. Both the revised and previous versions of this framework have previously been applied to understanding TCM and WM use in ethnic Chinese and other Asian populations ${ }^{4 ; 8}$, and use of TCM and other alternative treatment modalities in other populations ${ }^{10-12}$. According to the revised socio-behavioural model, use of health services is associated with personal health practices and population characteristics (which consist of predisposing, enabling, and need factors). Use of health services predicts outcomes, which are defined in the model as perceived and evaluated health status and patient satisfaction. The model also incorporates the broader environmental context, presenting the health care system and external environment as influencing population characteristics and health outcomes. As this study is primarily concerned with understanding TCM use and WM use we focus on those factors that are proximal to health services use within the socio-behavioural model: population characteristics and personal health practices.

Some population characteristics have been associated with use of TCM and WM in ethnic Chinese populations. Predisposing factors that influence health services use include demographic characteristics and beliefs about or attitudes towards health care. There is some evidence that, as in the general population, Chinese women living in the UK are more likely than Chinese men to consult WM GPs, and that the likelihood of GP consultations increases with age ${ }^{2}$. Female students were more likely than their male counterparts both to have a regular physician and to use CAM in a survey of Chinese students in the US ${ }^{13}$. Older people and those born in China tended to demonstrate more positive attitudes to TCM than younger people and those born in the UK in one focus group study ${ }^{14}$. However, demographic factors (education, age, gender) did not predict 
WM physician visits in a sample of immigrant Chinese elders in the US ${ }^{15}$. Indeed, surveys of Chinese students in Hong Kong suggest that TCM use might not be restricted to particular demographic groups within this sub-population: $85 \%$ of medical students ${ }^{16}$ $92 \%$ of nursing students ${ }^{17}$ and $96 \%$ of pharmacy students ${ }^{18}$ reported past TCM use. In comparison, amongst western-trained doctors in China older doctors had more positive attitudes to TCM than younger doctors ${ }^{19}$. Few studies have investigated the predictive value of relevant attitudes in understanding TCM and WM use. Hon's work suggests attitudes to TCM are predominantly positive or neutral in Hong Kong students, and that common justifications for using TCM include believing it to be more effective for a particular condition, finding WM to be ineffective for a condition, and believing TCM has fewer side-effects than WM ${ }^{16-18}$. Qualitative work also suggests that Chinese immigrants (to the UK, Canada and the US) who use TCM often view it as slower acting than WM, as more effective than WM for chronic conditions (but less effective for acute conditions - see below), and as better able to address the root cause(s) of health problems $1 ; 4 ; 14 ; 20$

Enabling factors can facilitate or inhibit use of health services, and include practical aspects of health care use, such as financial cost and language fluency, and social aspects such as the health care behaviours of significant others. Ability to speak English predicted GP visits amongst Chinese people living in the $\mathrm{UK}^{2}$. In addition, qualitative studies show that language and conceptual communication difficulties act as barriers to WM use and the financial cost of TCM consultations and medicines act as a barrier to TCM use ${ }^{1 ; 4 ; 21}$. Chinese students in Hong Kong commonly reported using 
TCM on recommendation from family and friends ${ }^{16-18}$, while social networks were also important for Chinese women in England talking about accessing TCM practitioners ${ }^{1}$.

Need is the third key individual-level determinant of health care use: people are more likely to use health services when they have an evaluated need (ie a formally diagnosed condition) or a perceived need (signs or symptoms of disease). Poor subjective health status has been associated with WM consultations in Chinese immigrants in the $\mathrm{UK}^{2}$ and the US ${ }^{22}$. As noted above, TCM is often thought to be better suited to treating chronic conditions while WM is thought to be more appropriate for acute conditions $1 ; 4 ; 14 ; 23$. In relation to specific conditions, Hon's student respondents in Hong Kong most often used TCM for upper respiratory tract infections ${ }^{16-18}$.

A person's reaction to a new episode of illness occurs in the context of their previous experiences of both illness and treatments: their previous health care practices will influence their future behaviour. Few studies have directly assessed how previous and current personal health practices influence TCM and WM use amongst Chinese immigrants, although some qualitative work suggests that people move between the two health care systems, using them in different ways according their own particular needs, characteristics, and circumstances ${ }^{1 ; 14 ; 24}$.

In summary, while the literature suggests that factors from the revised sociobehavioural model ${ }^{5}$ might be relevant to understanding Chinese students' use of TCM and WM in the UK, the model has not yet been examined in this particular population. Furthermore, previous studies of the health care practices of ethnic Chinese immigrants are of limited applicability given that our population of interest, international students, are more likely to be temporary migrants who are younger and more highly educated than 
other sub-groups. The present study thus uses the socio-behavioural model to explore the correlates of TCM and WM use in a group of ethnic Chinese international students in the UK. Given the lack of directly applicable previous literature, we combined our theoryinformed design with an exploratory, hypothesis-generating, approach. We expected that population characteristics and personal health practices would be associated with health services use, but do not present hypotheses concerning which specific factors might be associated with TCM and WM use in this population.

\section{$\underline{\text { METHODS }}$}

\section{$\underline{\text { Design and Procedure }}$}

This was an exploratory cross-sectional questionnaire study in which Chinese students at one British university completed measures of attitudes towards and use of TCM and WM. All ethnic Chinese international students at the host University were invited to take part (1053 such students were registered on 9 March 2007). Students were eligible to take part if they were ethnic Chinese and born and educated in China, Taiwan, Hong Kong, Singapore, or Malaysia. Invitations were placed on students' individualised home pages of the university community web portal, from where students could either follow a link to an online version of the questionnaire or contact the researchers for a paper version. The questionnaire took approximately 10 minutes to complete, and the introduction page stated that completion and return of the questionnaire indicated consent to participate in the study. The study was approved by the host institution's School of Psychology Ethical Committee.

\section{$\underline{\text { Materials }}$}


The questionnaire described below was presented in both English and Chinese, in order to improve its accessibility for the target population. The Chinese version was generated and back translated independently by two native Chinese speakers who are also fluent in English. The full questionnaire is available freely on request from the researchers.

\section{Predisposing factors}

We assessed demographic and attitudinal predisposing factors. Demographic and characteristics included age, gender, country of origin, University department, and duration of stay in the UK to date. Twenty three items measured attitudes to TCM and WM and were modified for this study from the Chinese-Western Medical Belief Scale 25;26. Modifications were required to improve the readability of some items in English and to eliminate double-barrelled items. Participants rated items on likert-type response scales ranging from 1 (strongly agree) to 5 (strongly disagree).

\section{Enabling factors}

We measured social and practical enabling factors. To assess the health behaviours of significant others participants were asked whether any family members had used WM and TCM in the past 12 months. One item measured participants' confidence in communicating in English. Eleven items assessed attitudes towards practical aspects (language, financial cost, and accessibility) of using WM and TCM in the UK need

Perceived need was operationalised as self-reported health status, and was assessed using items from the Health Survey for England ${ }^{27}$ : participants rated their overall health on a 5-point scale (Very Good, Good, Fair, Bad, Very Bad), and reported whether they had any 'long-standing illness, disability or infirmity'. 
Personal health practices

To assess previous personal health practices, participants were asked whether or not they had used WM and TCM in the past 12 months in the UK and in their last 12 months in their home country.

\section{$\underline{\text { Statistical Methods }}$}

Factor analysis (principal components with direct oblimin rotation) was carried out on the 34 attitude items (23 measuring attitudes to TCM and WM in general, 11 measuring attitudes to practical aspects of TCM and WM). This allowed us to assess which underlying constructs these individual items were measuring and reduced the number of predictor variables in our analyses hence maximising statistical power. Our sample size of 170 meets Gorsuch's guide of 5 cases per variable to carry out factor analysis ${ }^{28}$. An oblique rotation was chosen as there was no a priori reason to expect the underlying factors to be correlated. The solution was determined by considering eigenvalues, the scree plot, factor loadings including any cross-loadings, and the interpretability of the resulting factors ${ }^{29}$. Items with loadings of 0.5 and higher on a factor were interpreted as indicators of that factor, as this cut-off point produced clear, interpretable factors ${ }^{29}$. Items without loadings of 0.5 or higher on any factor were excluded from subsequent analyses.

Data were screened and 30 missing values were identified which were then imputed using the EM algorithm. Distributions of continuous variables were assessed and those that did not meet assumptions of Normality (age, accessibility of TCM, and financial cost of TCM) were transformed using median splits. 
Two separate multivariate logistic regression analyses were carried out to assess the predictors of TCM use and WM use by Chinese students in the UK. Forward stepwise selection (likelihood ratio) was used to determine which predictor variables entered each regression model. This statistical approach to logistic regression is appropriate for exploratory studies ${ }^{30}$.

\section{$\underline{\text { RESULTS }}$}

\section{$\underline{\text { Participants }}$}

184 students completed the questionnaire during Spring term 2007 (17\% of all Chinese students registered at the University). Fourteen participants of British nationality were excluded, leaving a sample of 170 participants who were aged between 18 and 39 $(\underline{\mathrm{M}}=23.9, \underline{\mathrm{SD}}=3.6)$. There were approximately equal numbers of men and women in the sample $(51 \%$ of participants $(\underline{n}=87)$ were female). The highest proportion of participants were from mainland China $(48 \%, \underline{n}=82)$ and Malaysia $(24 \%, \underline{n}=41)$, while others were from Taiwan $(14 \%, \underline{n}=24)$, Singapore $(7 \%, \underline{n}=12)$, and Hong Kong $(7 \%, \underline{n}=11)$. Students from across the University took part, with $47 \%(\underline{n}=80)$ from the arts and social sciences, $33 \%(\underline{n}=80)$ from engineering and physical sciences, and the remaining 20\% $(\underline{n}=34)$ from medicine and life sciences. Participants were confident $(\underline{n}=90,52 \%)$ or very confident $(\underline{n}=59,35 \%)$ communicating in English, with only $21(12 \%)$ stating they lacked confidence. They had been in the UK for between 2 and 96 months $(\underline{M}=27.2, \underline{S D}=20.2)$. Participants were generally healthy, with $74 \%(\underline{n}=125)$ rating their own health as good or very good, and $16 \%(\underline{n}=27)$ reporting a chronic illness. Table 1 shows that a high proportion of participants and their families used WM in the 12 months before coming to 
the UK while a notably smaller proportion of participants used TCM in the UK in the last 12 months.

Insert Table 1 Here

\section{Attitudes to Traditional Chinese and Western Medicine}

Five subscales measuring attitudes to TCM and WM with acceptable internal consistency were computed based on the results of the factor analysis (see Table 2). The subscales measured attitudes towards the effectiveness and safety of TCM, the effectiveness and rigour of WM, the accessibility of WM, the accessibility of TCM, and the financial cost of TCM. As Table 2 shows, the mean score was slightly above the mid-point on four of the scales, and slightly below the mid-point for the scale measuring attitudes towards the accessibility of TCM.

Insert Table 2 Here

\section{Use of Western Medicine in the UK}

The results of the stepwise logistic regression analysis of use of WM in the UK are summarised in Table 3. The model was a good fit to the data $\left(\chi^{2}(12)=52.04, p=.000\right)$ and the predictors explained approximately $37 \%$ of the variance in use of WM. The odds of using WM were decreased for participants who were from Singapore, and those who were studying arts/social sciences. The odds of using WM were increased for 
participants who were more confident in their English language skills, did have a chronic illness, used WM in their home country, and believed TCM is expensive in the UK.

Insert Table 3 Here

\section{Use of Traditional Chinese Medicine in the UK}

The results of the stepwise logistic regression analysis of TCM use in the UK are summarised in Table 4. The model was a good fit to the data $\left(\chi^{2}(6)=37.16, p=.000\right)$ and the predictors explained approximately $29 \%$ of the variance in TCM use. The odds of using TCM were decreased for participants who were studying medicine/life sciences. The odds of using TCM were increased for participants who used it in their home country, had more positive attitudes towards its effectiveness and safety, and believed it is expensive in the UK.

Insert Table 4 Here

\section{DISCUSSION}

This study has explored the patterns and predictors of the use of WM and TCM in a sample of Chinese students attending one British university. The majority of students used WM in both their home country and the UK. However, while the majority of them also used TCM in their home country a much smaller proportion used TCM in the UK. The statistical predictors were able to explain a modest proportion of the variance in both use of WM and use of TCM in this small sample. The study suggests further research is 
needed on the health care requirements and behaviours of this rapidly expanding subgroup of the Chinese population in the UK. In particular researchers should identify and explore ways to address the barriers that prevent Chinese students from accessing different health services in the UK.

WM use was associated with predisposing, enabling, and need factors, as well as personal health practices. TCM use was associated with predisposing factors, enabling factors, and personal health practices. This pattern of associations is consistent with the theoretical framework, Andersen's socio-behavioural model ${ }^{5}$. The specific predictors of use differed for each type of health care, and we offer tentative explanations for these findings below.

Participants were more likely to use WM if they were not from Singapore, if they were not studying arts or social sciences, if they were more confident in their English language skills, did have a chronic illness, used WM in their home country, and believed TCM is expensive in the UK. We cannot easily explain why participants who were not from Singapore were more likely to use WM, this might be an artefact of the uneven numbers of participants from different countries of origin. In relation to subject of study it is possible that arts and social science students might be more concerned with cultural values and less familiar with 'scientific' western medicine than those studying science and medical subjects, which could have led them to be less likely than other students to use WM. The association between use of WM and confidence in English skills echoes that found in a previous study ${ }^{2}$ and demonstrates that this issue is also relevant in the University setting. Therefore despite the fact that these students have demonstrated a certain level of English competency as a course pre-requisite they are reluctant to use 
WM if they have poorer English. The association between having a chronic illness and using WM supports the hypothesised relationship between evaluated need and health care use, but is less consistent with previous, qualitative, findings that TCM is often seen as more suitable for chronic illnesses than WM. This might be a consequence of the specific chronic illnesses present in our sample, but we had insufficient numbers of participants to investigate this. It makes sense that students who use WM in their home country are more likely to use WM when they come to the UK: further work is needed to explore whether this is because they have developed a preference for WM because of their experiences at home, or whether they are using long-term WM treatments both at home and in the UK.

Participants were more likely to use TCM if they were not studying medicine/life sciences, if they used it in their home country, had more positive attitudes towards its effectiveness and safety, and believed it is expensive in the UK. The higher use of TCM among non-medical students mirrors the higher use of WM among science students. We tentatively suggest that science students might be more familiar or comfortable with the 'scientific' approach in WM., and arts students might particularly value the socio-cultural and social identify aspects of TCM use. Green et al demonstrate how TCM use can be a way of maintaining and demonstrating a connection with one's culture of origin ${ }^{1}$ and this may explain TCM use by some students. Previous studies have shown that students report using TCM because they have positive attitudes towards its safety and effectiveness ${ }^{16-18}$; we have now shown that such positive attitudes are statistically associated with TCM use. The association between believing that TCM is expensive in the UK and both higher use of TCM and higher use of WM is puzzling. Our cross- 
sectional design may have obscured longitudinal patterns, perhaps students who use TCM find it to be expensive, while students who use WM do so because they perceive TCM to be prohibitively expensive.

This was a small, exploratory study that has generated tentative explanations about correlates of TCM and WM use that now need to be tested as hypotheses in a larger more representative sample of ethnic Chinese students in the UK. Many of our participants (45\%) chose to answer our questionnaire in Chinese, suggesting that future research in this area would benefit from making study materials available in both English and Chinese. More sophisticated study designs (e.g. prospective mixed methods studies) are also needed to further explore the reasons why these students appear to decrease their use of TCM when they come to the UK and the extent to which they are satisfied with the medical care they receive. The findings suggest that there are important individual differences in Chinese students' use of health care in the UK that cannot be fully understood using the current set of explanatory variables. It highlights perceived language skills as a barrier to using Western Medicine in this population suggesting that some Chinese students might benefit from increased support in this context. 


\section{REFERENCES}

(1) Green G, Bradby H, Chan A, Lee M. "We are not completely Westernised": Dual medical systems and pathways to health care among Chinese migrant women in England. Soc Sci Med 2006; 62(6):1498-1509.

(2) Sproston KA, Pitson LB, Walker E. The Use of Primary Care Services by the Chinese Population Living in England: examining inequalities. Ethnicity \& Health 2001; 6(3):189-196.

(3) Wu APW, Burke A, LeBaron S. Use of traditional medicine by immigrant chinese patients. Fam Med 2007; 39(3):195-200.

(4) Aroian KJ, Wu B, Tran TV. Health care and social service use among Chinese Immigrant elders. Res Nursing \& Health 2005; 28(2):95-105.

(5) Andersen R. Revisiting the behavioral model and access to medical care: does it matter? J Health Soc Behav 1995; 36:1-10.

(6) Andersen R, Newman JF. Societal and individual determinants of medical care utilization in the United States. Milbank Memorial Fund Quarterly 1973; 51:95124.

(7) Pescosolido B. Beyond Rational Choice: The Social Dynamics of How People Seek Help. American Journal of Sociology 1992; 97(4):1096-1138.

(8) Miltiades HB, Wu B. Factors affecting physician visits in Chinese and Chinese immigrant samples. Soc Sci Med 2008; 66(3):704-714.

(9) Jang Y, Kim G, Chiriboga DA. Health, Healthcare Utilization, and Satisfaction with Service: Barriers and Facilitators for Older Korean Americans. J Am Ger Soc 2005; 53(9):1613-1617. 
(10) Upchurch DM, Burke A, Dye C, Chyu L, Kusunoki Y, Greendale GA. A Sociobehavioral Model of Acupuncture Use, Patterns, and Satisfaction Among Women in the United States, 2002. Women's Health Issues 2008; 18(1):62-71.

(11) Fouladbakhsh JM, Stommel M, Given BA, Given CW. Predictors of use of complementary and alternative therapies among patients with cancer. Oncology Nursing Forum 2008; 32(6):1115-1122.

(12) Kelner M, Wellman B. Health care and consumer choice: Medical and alternative therapies. Soc Sci Med 1997; 45(2):203-212.

(13) Ray-Mazumder S. Role of gender, insurance status and culture in attitudes and health behavior in a US Chinese student population. Ethnicity and Health 2001; 6(3/4):197-209.

(14) Prior L, Chun PL, Huat SB. Beliefs and accounts of illness. Views from two Cantonese-speaking communities in England. Sociology of Health \& Illness $2000 ; 22(6): 815-839$.

(15) Miltiades HB, Wu B. Factors affecting physician visits in Chinese and Chinese immigrant samples. Soc Sci Med 2008; 66(3):704-714.

(16) Hon EKL, Leung TF, Tse HM, Lam LN, Tam KC, Chu KM et al. A survey of attitudes to traditional Chinese medicine among Chinese medical students. American Journal of Chinese Medicine 2005; 33(2):269-279.

(17) Hon EKL, Twinn SF, Leung TF, Thompson DR, Wong Y, Fok TF. Chinese nursing students' attitudes toward traditional Chinese medicine. Journal of Nursing Education 2006; 45(5):182-185. 
(18) Hon EKL, Lee K, Tse HM, Lam LN, Tam KC, Chu KM et al. A survey of attitudes to traditional Chinese medicine in Hong Kong pharmacy students. Complement Ther Med 2004; 12(1):51-56.

(19) Harmsworth K, Lewith GT. Attitudes to traditional Chinese medicine amongst Western trained doctors in the People's Republic of China. Soc Sci Med 2001; 52:149-153.

(20) Zhang J, Verhoef MJ. Illness management strategies among Chinese immigrants living with arthritis. Soc Sci Med 2002; 55(10):1795-1802.

(21) Zhang J, Verhoef MJ. Illness management strategies among Chinese immigrants living with arthritis. Soc Sci Med 2002; 55(10):1795-1802.

(22) Miltiades $\mathrm{HB}, \mathrm{Wu}$ B. Factors affecting physician visits in Chinese and Chinese immigrant samples. Soc Sci Med 2008; 66(3):704-714.

(23) Zhang J, Verhoef MJ. Illness management strategies among Chinese immigrants living with arthritis. Soc Sci Med 2002; 55(10):1795-1802.

(24) Zhang J, Verhoef MJ. Illness management strategies among Chinese immigrants living with arthritis. Soc Sci Med 2002; 55(10):1795-1802.

(25) Chan MF, Mok E, Wong YS, Tong TF, Day MC, Tang CKY et al. Attitudes of Hong Kong Chinese to traditional Chinese medicine and Western medicine: survey and cluster analysis. Complement Ther Med 2003; 11(2):103-109.

(26) Liang CC. The development and examination of the Chinese-Western beliefs scale. Nursing Research (in Chinese) 1999; 7(5):445-458.

(27) The Health Survey for England. http://qb soc surrey ac uk/surveys/hse/04iqhse pdf [accessed Jan. 30 2007] 
(28) Gorsuch RL. Factor Analysis. 2nd ed. Hillsdale, NJ: Erlbaum; 1983.

(29) Tabachnick BG, Fidell LS. Using Multivariate Statistics. 4 ed. Boston: Allyn and Bacon; 2001.

(30) Field A. Discovering statistics using SPSS for Windows. London: Sage; 2000. 
Table 1.

Proportion of Students Reporting Using Western and Traditional Chinese Medicine

\begin{tabular}{llcll}
\hline & \multicolumn{2}{l}{ Use of Western Medicine } & \multicolumn{2}{l}{ Use of Traditional Chinese Medicine } \\
& \multicolumn{2}{l}{ in past 12 months } & in past 12 months \\
\cline { 2 - 5 } & $\%$ & $\mathrm{n}$ & $\%$ & $\mathrm{n}$ \\
\hline Self in UK & 70 & 119 & 25 & 42 \\
Self in home country & 87 & 148 & 68 & 116 \\
Family members & 95 & 162 & 84 & 142 \\
\hline
\end{tabular}


Table 2.

Factor Loadings from Pattern Matrix of Items Assessing Attitudes to Western Medicine and Traditional Chinese Medicine

Item Component $^{1}$

\begin{tabular}{llll}
\hline 1 & 2 & 3 & 4
\end{tabular}

Compared with western medicine, TCM is more effective for

.34

$-.21$

long-standing diseases

TCM treats the cause of illness

$.64-.15$

.14

Compared with western medicine, TCM has fewer side effects

Compared with western medicine, TCM causes less digestive

side effects

TCM can cure diseases

$.61 \quad-.12$

TCM can promote health

$\begin{array}{llll}.83 & .18 & -.10 & -.18\end{array}$

TCM can have a restorative effect which can promote health

over time

TCM can improve one's constitution

Compared with TCM, western medicine can diagnose illness

more accurately

Compared with TCM, western medicine has better health care

facilities available

Compared with TCM, the overall quality of western medicines

is better

Compared with TCM, the process of education and training in -.14
.72

.84 
western medicine is more rigorous

Compared with TCM, western medicine has a more scientific $\quad \begin{array}{llll}\text { C.13 } & \mathbf{5 3} & -.30\end{array}$

approach to illness

Compared with TCM, western medicine acts more quickly

.11

.58

$.16-.16$

Compared with TCM, western medicine is more effective for

.63

$.17-.13$

acute illness

Consulting a TCM doctor in the UK is too expensive for me

Chinese herbs in the UK are too expensive for me

It has been/would be easy for me to find a TCM doctor in the

$-.14$

.21

$-.13$

.64

.28

UK

It has been/would be easy for me to find a trustworthy TCM

doctor in the UK

It has been/would be easy for me to find a trustworthy western

medicine doctor in the UK

It has been/would be easy for me to understand the language

that western medicine doctors use in the UK

It has been/would be easy for me to understand the ideas that $\quad .11$

western medicine doctors use in the UK

\begin{tabular}{lccccc}
\hline Mean & 3.67 & 3.77 & $\mathbf{3 . 4 2}$ & 2.41 & 3.57 \\
Standard deviation & 0.69 & 0.62 & $\mathbf{0 . 7 9}$ & 0.78 & 0.72 \\
Cronbach's Alpha & .87 & .82 & .75 & .61 & .81
\end{tabular}

Note. Loadings $<.1$ are omitted and loadings $>.5$ are highlighted in bold typeface. 
${ }^{1}$ Component 1 = Effectiveness and Safety of TCM, 2 = Effectiveness and Rigour of WM, $3=$ Accessibility of WM, 4 = Accessibility of TCM, $5=$ Financial Cost of TCM. 
Table 3.

Summary of Stepwise Logistic Regression to Predict use of Western Medicine by

Chinese Students in the UK

\begin{tabular}{llllll}
\hline Predictors & $\underline{\mathrm{SE}} \quad$ Wald & OR & $95 \% \mathrm{CI}$ \\
\cline { 4 - 5 } & & & &
\end{tabular}

$\underline{\text { Predisposing Factors }}$

Country of origin: Mainland China

(reference category)

$10.716^{*}$

$\begin{array}{lrrrrrr}\text { Hong Kong } & 1.189 & 1.176 & 1.022 & 3.283 & .328 & 32.896 \\ \text { Taiwan } & .613 & .599 & 1.046 & 1.846 & .570 & 5.973 \\ \text { Malaysia } & -.544 & .644 & .714 & .580 & .164 & 2.050 \\ \text { Singapore } & -2.462 & .900 & 7.483^{* *} & .085 & .015 & .498\end{array}$

Course engineering/science

(reference category)

Arts/social sciences

$\begin{array}{llllll}-1.295 & .506 & 6.545^{*} & .274 & .102 & .739\end{array}$

Medicine/life sciences

$\begin{array}{llllll}.454 & .775 & .344 & 1.575 & .345 & 7.189\end{array}$

Enabling Factors

Language confidence low

(reference category)

Language confidence

medium

$\begin{array}{llllll}1.132 & .575 & 3.868^{*} & 3.101 & 1.004 & 9.580\end{array}$

$\begin{array}{lllllll}\text { Language confidence high } & 1.989 & .720 & 7.628 * * & 7.309 & 1.782 & 29.981\end{array}$

$\begin{array}{llllllll}\text { Financial cost of TCM } & .989 & .425 & 5.417 * & 2.689 & 1.169 & 6.186\end{array}$ 
$\underline{\text { Need }}$

Good subjective health$$
-.907 \quad .520
$$

520

3.050

$.404 \quad .146$

1.117

No chronic illness

$-1.599$

.745

$4.600 *$

.202

$.047 \quad .871$

$\underline{\text { Personal Health Practices }}$

Use WM in home country

$$
2.098
$$

$.61411 .669 * *$

8.151

2.446

27.167

Constant

.265

1.172

.051

1.304

Note. Coefficients for final step only are shown.

Variables that did not meet the inclusion criteria: gender, age, length of time lived in

UK, use of TCM in home country, family use of TCM, family use of WM, effectiveness

and safety of TCM, effectiveness and rigour of WM, accessibility of WM, accessibility of TCM.

$* \mathrm{p}<.05 ; * * \mathrm{p}<.01$ 
Table 4.

Summary of Stepwise Logistic Regression to Predict use of Traditional Chinese

Medicine by Chinese Students in the UK

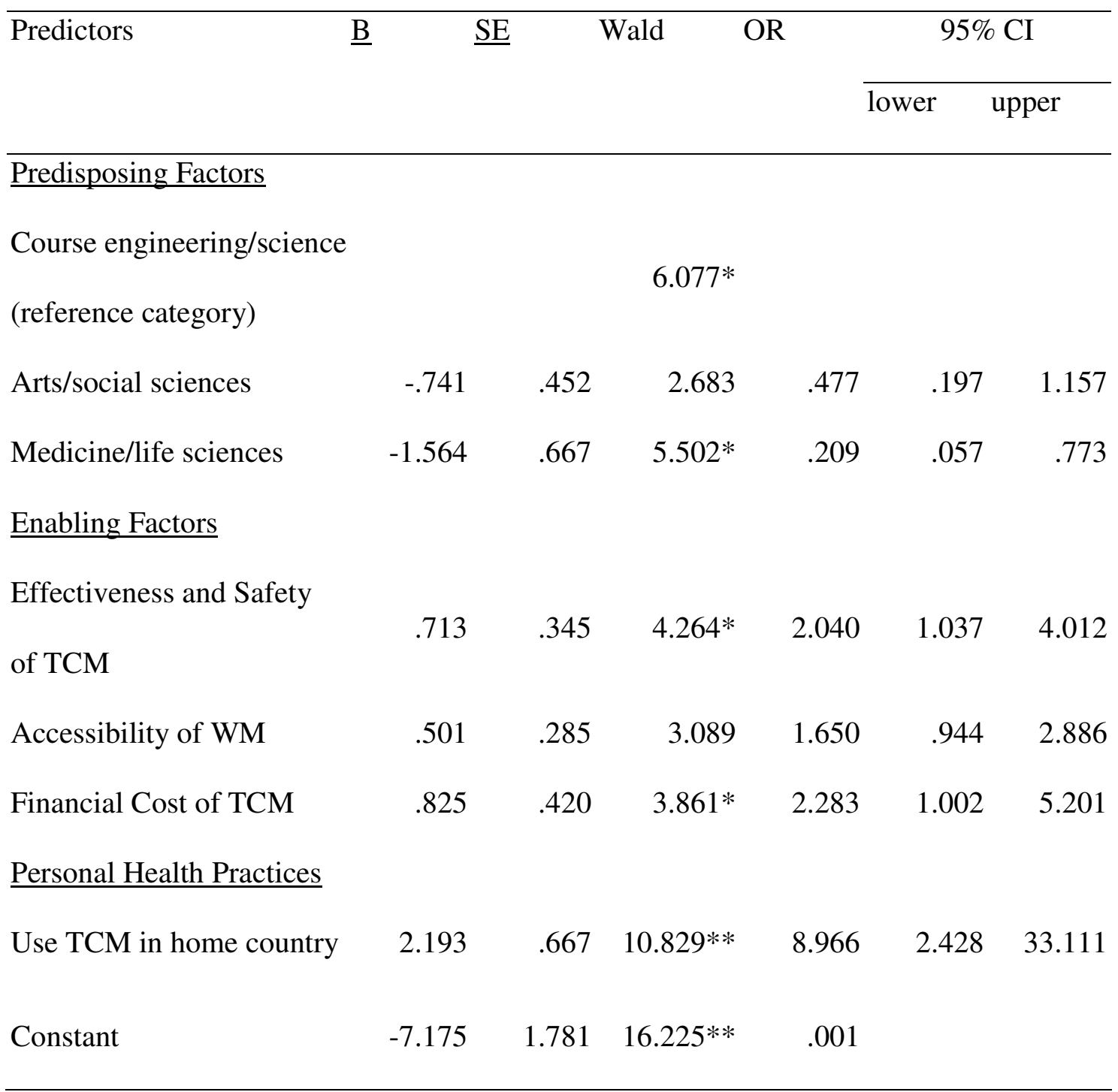

Note. Coefficients for final step only are shown.

Variables that did not meet the inclusion criteria: gender, age, country of origin, length

of time lived in the UK, language confidence, subjective health status, presence of chronic illness, use of WM in home country, family use of WM, family use of TCM, effectiveness and rigour of WM, accessibility of TCM. 
${ }^{*} \mathrm{p}<.05 ; * * \mathrm{p}<.01$ 